Rev. Biol. Trop., 48(2/3): 487-494, 2000

www.ucr.ac.cr www.ots.ac.cr www.ots.duke.edu

\title{
Espectro trófico de la tilapia Oreochromis aureus (Perciformes: Cichlidae) en la presa Infiernillo, Michoacán-Guerrero, México
}

Ma. de Lourdes Jiménez-Badillo ${ }^{1}$ y Marta Rocío Nepita-Villanueva ${ }^{2}$

1 Universidad Autónoma Metropolitana Unidad Iztapalapa. División de Ciencias Biológicas y de la Salud. Departamento de Hidrobiología. A.P. 55-535. C.P. 09340. México, D.F. Tel. 52 (5) 8-04-47-40. Fax 52 (5) 8-04-47-38. Correo electrónico:mljb@xanum.uam.mx

2 Centro de Investigación en Alimentación y Desarrollo. Laboratorio de Manejo Ambiental. Sábalo Cerritos s/n, Estero del Yugo. A.P. 711. C.P. 82010. Mazatlán, Sinaloa. México. Tel. 52 (69) 88-01-57. Fax 52 (69) 88-01-59. Correo electrónico: rnepita@ victoria.ciad.mx

Recibido 4-VIII-1999. Corregido 20-III-2000. Aceptado 23-III-2000.

\begin{abstract}
The trophic spectrum of tilapia Oreochromis aureus (Steindachner 1864), was determined by stomach content analysis in 153 organisms collected during 1993 in Michoacán-Guerrero, México. The feeding status of the fish at the time of observation was evaluated by the examination of fat surrounding the gut, gastric replection and the condition coefficient. The quantitative evaluation of the food items was carried out by the combination of the following analysis: Frequency of Occurrence, Volumetric Method, Volumetric Mean Index and Relative Importance Index. Diet consisted of: detritus and vascular plant residues as a primary food; unicellular algae as secondary food; and remains of insects and fish, graminean seeds, filamentous algae, cladocerans, ostracods, rotifers and copepods as occasional food. We detected a difference in food preferences between juveniles and adults and a variation in the consumption proportions of some food items during the rainy and dry seasons. Thus, we concluded that $O$. aureus is an omnivorous species with preference for detritus and vascular plant remain. Feeding seems to be determined by the abundance of food items found in the habitat and adults showed a trend to eat only detritus. Feeding does not seem to be a limiting factor in the development of the tilapia in Infiernillo dam.
\end{abstract}

Key words: Oreochromis aureus, tilapia, food analysis, Infiernillo dam, México.

La presa Adolfo López Mateos "Infiernillo", constituye el principal embalse productor de tilapia Oreochromis aureus en México. Desde que se inició la pesquería en 1970 ha sido el sustento de alrededor de 44467 habitantes (Anónimo 1998), además de ocupar laboralmente un número aún mayor entre pescadores, intermediarios, fileteadores y comerciantes finales. Los mayores niveles de producción registrados en la historia de la presa se remontan a 1987 cuando alcanzó 18953 ton; desde entonces ha existido un continuo descenso al grado que actualmente solo se registran 5413 ton (Anónimo 1998).
La información científica generada alrededor de la presa ha sido aislada. Se han hecho estudios hidrológicos (Cortés y Arredondo 1976, Bernal 1984, Juárez 1989, Jiménez et al. 1994), sobre parasitismo (Rosas 1976), crecimiento (Guzmán 1994, Palacios 1995), dinámica poblacional (Bernal 1984, Jiménez 1999), reproducción (Palacios 1995, Jiménez 1999) y pesquería (Romero y Orbe 1988, Juárez 1989, Jiménez 1999), pero el tema alimentario no ha sido abordado.

Se planteó una evaluación integral de la pesquería que permitiera detectar los factores causantes del descenso en la producción. En 
este artículo se presentan sólo los resultados de la evaluación alimentaria. Este aspecto ocupa un lugar relevante, al detectar las oportunidades de desarrollo que la tilapia tiene en este entorno, así como comprender el papel que desempeña dentro del ecosistema.

Se incluye además la variación que la alimentación puede presentar en función de las fluctuaciones cíclicas, como: nivel de la presa, época del año o etapa de desarrollo de las tilapias.

\section{MATERIALES Y MÉTODOS}

La presa Infiernillo, localizada en la cuenca del Río Balsas, entre los límites de los estados de Michoacán y Guerrero, México, se creó en 1963 para la generación de energía eléctrica. Su longitud máxima es de $120 \mathrm{~km}$ y tiene una profundidad media de $35 \mathrm{~m}$. Su superficie inundada en los niveles de máxima y mínima operación, fluctúa entre 34600 y 14100 ha (Juárez 1989). Los ríos que la surten son el Tepalcatepec por el Norte y Balsas por el Este. Como actividad secundaria se le emplea en la producción de tilapia, carpa y bagre.

El espectro trófico de la tilapia $O$. aureus, que representa el $90 \%$ de la producción en la presa, se determinó a partir de muestreos realizados durante marzo, mayo, julio, septiembre y noviembre de 1993, en Churumuce, Nuevo Centro, Platanito y Pinzandarán. Cada muestra consistió de 30 peces, seleccionados al azar y colectados con una red tipo chinchorro de $50 \mathrm{~m}$ de longitud, caída de $3 \mathrm{~m}$ y una abertura de malla de $1 \mathrm{~cm}$. De cada organismo se registró su longitud estándar, peso y sexo. Estómagos e intestinos fueron fijados en formol al 7\%.

El estado de alimentación se evaluó mediante la proporción de grasa rodeando el estómago e intestino y la replexión gástrica de acuerdo a Casas y Benítez (1976) y el coeficiente de condición con base en Bagenal y Tesch (1978).

Los contenidos estomacales de cada ejemplar, fueron examinados con ayuda de un mi- croscopio estereoscópico y los elementos alimentarios determinados con un microscopio óptico, hasta el menor nivel taxonómico posible con base en Needham (1962), Barnes (1977), Pennak (1978) y Ortega (1984).

La evaluación cuantitativa de los elementos alimentarios encontrados, se realizó mediante los métodos Volumétrico (V), Frecuencia de Aparición (FA) (Windell y Bowen 1978, Hyslop 1980), el Indice Medio Volumétrico (IMV) (Saiki 1976, modificado por Martínez 1983), cuyo menor valor numérico indica mayor importancia volumétrica y el Indice de Importancia Relativa (IIR) (Pinkas et al. 1971 modificado por Yañez-Arancibia et al. 1976), que establece una relación entre la cantidad y el volumen de cada categoría alimentaria expresadas como porcentaje.

A través del diagrama trófico combinado, que relaciona los métodos e índices anteriores, fue posible definir, los elementos alimentarios preferentes (40 a 100\%), secundarios (10 a $40 \%$ ) y ocasionales ( 0 a $10 \%)$, según el criterio de Yañez et al. (1976).

Con respecto a la variación en la dieta de $O$. aureus, ésta se evaluó a través de la comparación de los diagramas tróficos obtenidos al considerar el estado de madurez (juvenil-adulto), utilizando como criterio de separación, la talla de primera madurez, establecida en $170 \mathrm{~mm}$ de longitud estándar (Jiménez et al. 1997) y en función de la época del año, considerando época de secas de noviembre a mayo y época de lluvias de junio a octubre, que coinciden con las épocas de vaciado y llenado de la presa, respectivamente.

\section{RESULTADOS}

Del total de ejemplares analizados, $60 \%$ resultaron ser machos y $40 \%$ hembras. Sus tallas fluctuaron entre 37 y $255 \mathrm{~mm}$ de longitud estándar y sus pesos entre 2 y 431 g. El $8 \%$ de los ejemplares fueron inmaduros, $19.4 \%$ se encontraron en maduración, $71.3 \%$ maduros y $1.2 \%$ desovados.

El análisis de replexión gástrica determinó un $85.6 \%$ de los ejemplares con estómagos 
completamente llenos, un $5.2 \%$ con estómagos vacíos en marzo y mayo que coinciden con la época de secas, un $3.9 \%$ con estómagos casi llenos $(1 / 2-3 / 4)$ y un $5.2 \%$ con estómagos casi vacíos (1/4 - 1/3). También se observaron $39.2 \%$ de los ejemplares sin grasa alrededor de su intestino, $2.6 \%$ con grasa abundante en marzo y julio, $29.4 \%$ con mucha grasa en mayo y $28.7 \%$ con poca grasa. El coeficiente de condición de la tilapia fluctuó entre 0.02 y 0.38 a través del año. Los valores mayores se presentaron en la época de lluvias (Fig. 1) y coincidieron con los picos de desove señalados por Jiménez et al. (1997) en junio y octubre.

Se identificaron 11 elementos alimentarios diferentes en los estómagos de las tilapias, cuyos porcentajes estimados por los diferentes métodos, se presentan en el Cuadro 1.

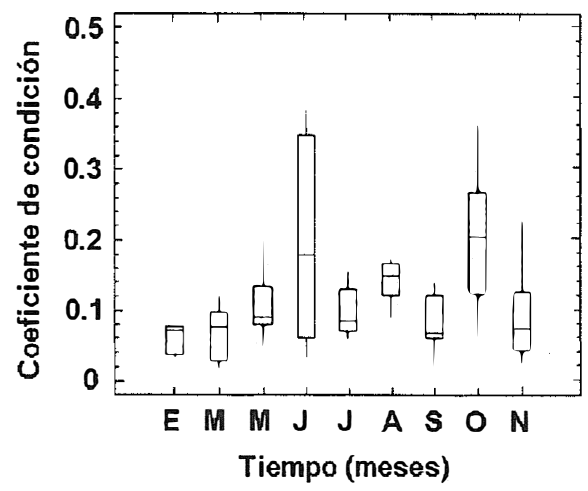

Fig. 1. Coeficiente de condición de Oreochromis aureus a través del tiempo.

Fig. 1. Condition coefficient for Oreochromis aureus through time.

\section{CUADRO 1}

Composición de la dieta alimentaria de Oreochromis aureus en la presa Infiernillo

Diet composition of Oreochromis aureus at the Infiernillo dam

\section{Contenido estomacal \\ (145 ejemplares)}

Detritus

Algas unicelulares

Algas filamentosas

Restos plantas vasculares

Semillas de gramíneas

Cladóceros

Ostrácodos

Rotíferos

Restos de Insectos

Restos de Peces

Copépodos

$\begin{array}{crcr}\text { FA \% } & \text { V \% } & \text { Método } & \\ & & \text { IIR \% } & \text { IMV } \\ 93.1 & 69.4 & 64.6 & 1.9 \\ 14.5 & 1.5 & 0.21 & 9.8 \\ 5.5 & 0.8 & 0.04 & 10.5 \\ 53.1 & 24.9 & 13.2 & 5.9 \\ 9.7 & 0.12 & 0.01 & 10.2 \\ 7.6 & 0.11 & 0.01 & 10.4 \\ 5.5 & 0.14 & 0.01 & 10.6 \\ 1.4 & 0.01 & 0.00 & 10.9 \\ 9.7 & 0.63 & 0.06 & 10.2 \\ 6.9 & 2.40 & 0.17 & 10.4 \\ 0.7 & 0.001 & 0.0007 & 11.2\end{array}$

$$
\begin{aligned}
& \text { FA = Frecuencia de Aparición } \\
& \text { V = Método Volumétrico } \\
& \text { IIR = Indice de Importancia Relativa } \\
& \text { IMV = Indice Medio Volumétrico }
\end{aligned}
$$

La categoría detritus incluye materia orgánica no identificada y una pequeña proporción de arena. Dentro de las algas unicelulares predominaron diatomeas (división Chrysophyta) y en menor grado euglenas (Euglenophyta); entre las algas filamentosas se presentaron algas ver- des y algas verdeazules (Divisiones Chlorophyta y Cyanophyta, respectivamente). Dentro del Subphylum Crustacea se identificaron cladóceros (Orden Cladocera) y ostrácodos (Clase Ostracoda), sólo en un ejemplar de tilapia se encontraron copépodos (Subclase Copépoda). 
También se encontraron rotíferos (Phylum Rotífera). Los restos de peces incluyeron escamas, espinas y huevecillos de tilapia. Entre los restos de insectos se identificaron efemerópteros (Orden Ephemeroptera).

Del total de estómagos analizados, en un $34 \%$ se encontró sólo detritus. De hecho, los alimentos preferentes de $O$. aureus fueron el detritus y los restos de plantas vasculares. Los demás elementos alimentarios quedaron comprendidos dentro de la categoría de alimento ocasional (Fig. 2), ocupando un mismo nivel de importancia volumétrica según lo indicó el índice medio volumétrico.

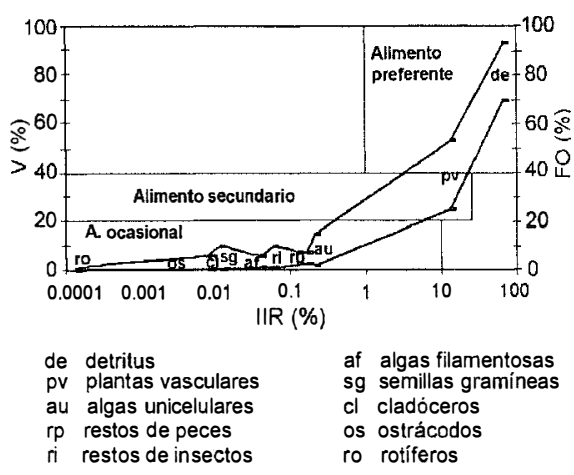

Fig. 2. Diagrama trófico combinado de Oreochromis aureus en la presa Infiernillo

Fig. 2. Combined trophic diagram for Oreochromis aureus at the Infiernillo dam

Al comparar las preferencias alimentarias de los organismos juveniles y adultos, se encontró que éstos últimos se alimentan casi exclusivamente de detritus. Los restos de plantas vasculares pasan de un $15.5 \%$ de importancia relativa en los juveniles a $0.007 \%$ en los adultos. El resto de los elementos alimentarios fueron sólo ocasionales y registrados en bajas proporciones (Fig. 3).

En cuanto a la variación mensual en la composición de la dieta de $O$. aureus, los
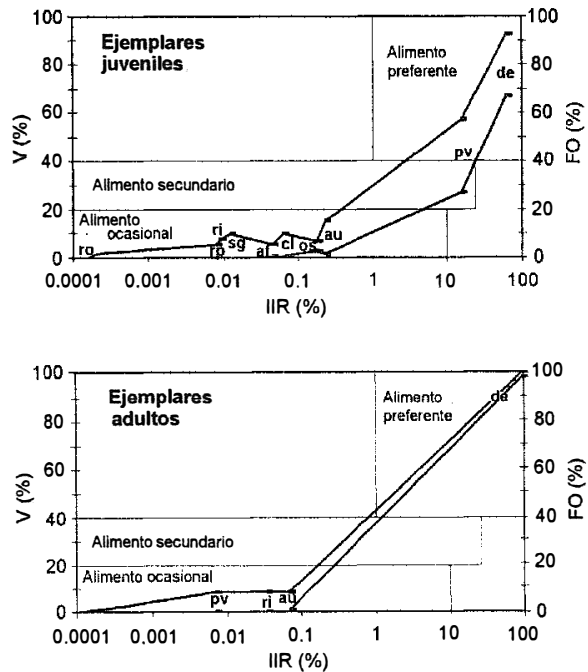

$$
\begin{array}{ll}
\text { de detritus } & \text { af algas filamentosas } \\
\text { pv restos plantas vasculares } & \text { sg semillas gramineas } \\
\text { au algas unicelulares } & \mathrm{cl} \text { cladoceros } \\
\text { rp restos peces } & \text { os ostrácodos } \\
\text { ri restos insectos } & \text { ro rotiferos }
\end{array}
$$

Fig. 3. Preferencia alimentaria en juveniles y adultos de Oreochromis aureus

Fig. 3. Food preferences of juveniles \& adults of Oreochromis aureus.

cambios más notables se presentaron en las plantas vasculares, que después de ocupar una posición preferente en marzo, su baja proporción en mayo las ubicó como alimento secundario, para volver a incrementar en julio y septiembre. En éstos meses, las algas unicelulares y filamentosas que en marzo estaban ausentes, constituyeron un alimento ocasional. La proporción de las algas unicelulares incrementó en septiembre y noviembre cuando llegó a constituir un alimento secundario junto con las plantas vasculares. El detritus siempre fue un alimento preferente (Fig. 4).

A pesar de estos cambios mensuales, la tendencia general es a mantener una proporción similar de detritus y plantas vasculares tanto en época de secas como de lluvias. Los 
ostrácodos, restos de peces y de insectos tienden a disminuir en época de lluvias y la proporción de algas unicelulares y filamentosas a
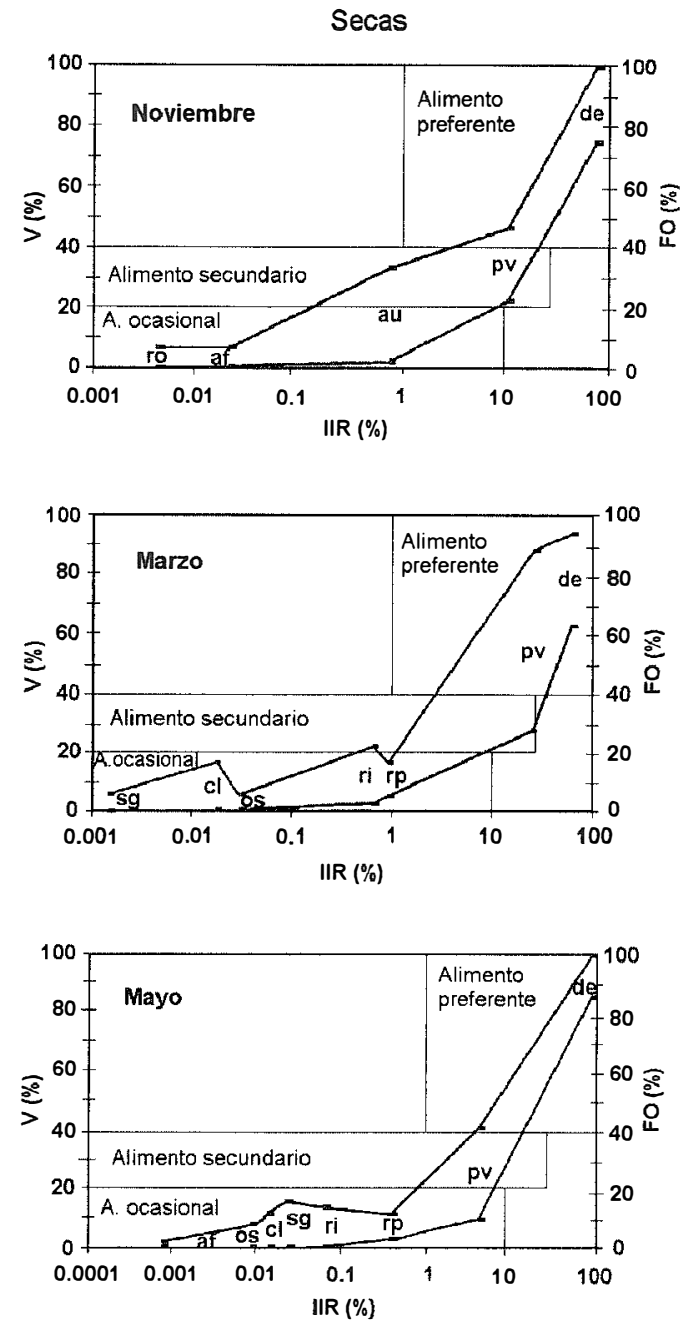

incrementar notablemente. En la época de estiaje es mayor la proporción de semillas de gramíneas y cladóceros (Fig. 4).
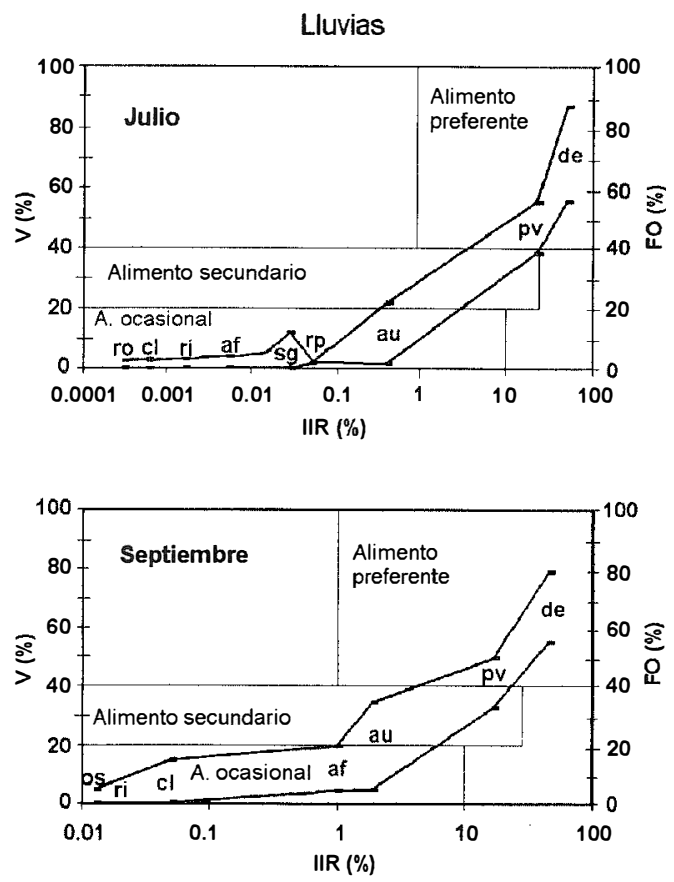

de detritus

af algas filamentosas

au algas unicelulares

restos de peces

g semillas de gramineas

cl cladóceros

os ostrácodos

ro rotiferos

ri restos de insectos pv restos plantas vasculares

Fig. 4. Variación en la dieta de Oreochromis aureus de acuerdo a la época del año

Fig. 4. Diet variation of Oreochromis aureus through the year

\section{DISCUSIÓN}

La dieta de los peces es un reflejo de los alimentos que están disponibles en el ambiente (Wootton 1990) y de alguna manera, su disponibilidad puede determinar la preferencia alimentaria. En el presente caso, la preferencia hacia detritus y restos de plantas vasculares, obedece a su abundancia en el medio, favorecida por la inundación de los cultivos temporales que se establecen en la ribera de la presa durante la época de secas y que en el año en que se realizaron los muestreos, no fueron cosechados en su totalidad, 
por las lluvias tempranas, además de la vegetación natural que se implanta en las áreas desecadas que constituyen al descomponerse, un importante aporte de materia orgánica. $\mathrm{Al}$ mismo tiempo, se tiene el acarreo de los ríos Tepalcatepec y Balsas, que desembocan en la presa y aportan importantes volúmenes de materia orgánica al sistema (Juárez 1989, Jiménez et al. 1994).

En otros embalses de México e Israel, se ha caracterizado a $O$. aureus como fitoplanctófaga (Morales 1974), consumidora de fitoplancton, zooplancton y zoobentos (Spataru y Zorn 1978a, 1978b), omnívora (Mc Bay 1961) y omnívora con predominio de organismos del zooplancton (Salvadores 1980, Salvadores y Guzmán 1983). Wootton (1990) reporta la capacidad de los cíclidos típicamente zooplanctófagos, insectívoros o detritívoros de cambiar su consumo hacia diatomeas, cuando éstas son muy abundantes. La capacidad de la tilapia para alimentarse de un amplio espectro trófico, le confiere la posibilidad de consumir otros elementos alimentarios cuando los preferentes son escasos. Esta versatilidad contribuye a su adaptación en ambientes cambiantes.

Respecto a la preferencia hacia ciertos elementos alimentarios, en función del estado fisiológico del pez, Mc Bay (1961), señaló que en peces menores de $150 \mathrm{~mm}$ se presentaron grandes concentraciones de zoo-organismos, mientras que en organismos mayores fue más alta la proporción de vegetales. En el presente estudio, se detectó una diferencia de preferencia alimentaria entre juveniles y adultos, dirigida en los últimos casi exclusivamente hacia el detritus, lo que puede deberse a que durante esta etapa en que se reproducen y pasan mucho tiempo cerca del nido, su alimentación se ve restringida a la disponibilidad de detritus en el medio. Por el contrario, los organismos juveniles presentaron una mayor variedad de elementos alimentarios en sus estómagos.

Por otra parte, los cambios estacionales en la disponibilidad de alimentos, pueden ser causados por cambios en la disponibilidad del hábitat, como sucede en la presa Infiernillo, cuyo nivel fluctúa mensualmente por el uso que se da al agua para la generación de energía eléctrica. El máximo descenso en su nivel es de alrededor de $22 \mathrm{~m}$. La máxima profundidad detectada en la presa cerca de la cortina fue de 60 $\mathrm{m}$, en la entrada de los ríos de 22 y $12 \mathrm{~m}$ y hacia la parte central de la presa de $40 \mathrm{~m}$, por tanto, este descenso representa una reducción del 37 al $67 \%$ en su profundidad. El efecto más evidente se presenta en sentido horizontal ya que durante la etapa de vaciado de la presa llegan a quedar al descubierto entre 10 y $15 \mathrm{~km}$, que incluso son aprovechados para la siembra durante este tiempo, lo que obliga a las tilapias a migrar hacia la cortina (Jiménez 1999).

Esta etapa de descenso en el nivel de la presa (enero a junio) coincide con la época de secas en la que la competencia interespecífica por alimento y espacio puede acentuarse. Los contenidos estomacales de la tilapia revelaron que en esta época la materia vegetal sobrepasó ligeramente a la materia animal.

El acarreo de nutrientes y la inundación de terrenos de cultivo, pueden generar una mayor cantidad y calidad de alimento. En la época de lluvias, que coincide con la inundación de la presa, en que el florecimiento y disponibilidad del fitoplancton es elevado, así como la presencia de restos vegetales, se presentó una tendencia hacia el predominio de consumo de elementos vegetales. También se observó una tendencia a incrementar el coeficiente de condición de la tilapia en ésta época, lo que indica que su estado físico se vio favorecido en este período.

Más de una tercera parte de los organismos analizados, no presentaron grasa alrededor de su estómago e intestino. Esto puede estar indicando que su metabolismo acelerado por el continuo proceso de reproducción que aquí se presenta, evita que se produzca la acumulación de grasas.

Las especies que conviven en la presa con $O$. aureus y con las cuales puede existir sobreposición de dietas son la carpa común (Cyprinus carpio) y la tilapia mosambica (Oreochromis mossambicus) que se alimentan de plancton, macrófitas, bentos y detritus, así como la tilapia nativa (Ciclasoma istlanum), que comparte algunos de los elementos alimentarios 
con $O$. aureus y que ha sido casi completamente desplazada por esta especie, debido a la competencia en las áreas de postura pues tienen hábitos reproductivos similares (Rosas 1976). Sin embargo, por la baja abundancia en que se encuentran estas especies (menos del 10 $\%)$, no representan una amenaza para la especie en estudio.

Esta evaluación permitió detectar que la alimentación no es un factor que limite el desarrollo de la tilapia en Infiernillo. Existe evidencia para suponer que la disminución en la abundancia obedece a problemas de sobrepesca y alteración de la estructura genética y poblacional de la tilapia (Jiménez, 1999).

$O$. aureus es una especie omnívora que incluye en su espectro trófico detritus y plantas vasculares con preferencia. Las algas unicelulares se consideran un alimento secundario y los demás elementos alimentarios son ocasionales.

\section{AGRADECIMIENTOS}

Agradecimos a Claudio Osuna Paredes y Casimiro Ramírez Camarena por su valiosa colaboración en los muestreos. A Irene Barriga por la revisión del manuscrito y al Instituto Nacional de la Pesca por financiar esta investigación.

\section{RESUMEN}

Se determinó el espectro trófico de la tilapia Oreochromis aureus (Steindachner 1864), a través del análisis del contenido estomacal de una muestra de 153 especímenes recolectados durante 1993. El estado de alimentación se evaluó por la proporción de grasa alrededor del estómago e intestino, la replexión gástrica y el coeficiente de condición. La evaluación cuantitativa del alimento, se realizó a través de los métodos Volumétrico (V) y Frecuencia de Aparición (FA) y los Indices Medio Volumétrico (IMV) e Importancia Relativa (IIR), los cuales se combinaron para determinar el espectro trófico de la tilapia. Se determinó que su dieta en la presa Infiernillo está constituida por: detritus y plantas vasculares como alimentos preferentes; algas unicelulares como alimento secundario y peces, insectos, semillas de gramíneas, algas filamentosas, cladóceros, ostrácodos, rotíferos y copépodos, como alimentos ocasionales. Se detectaron diferencias alimentarias entre juveni- les y adultos y variaciones en el consumo de elementos alimentarios en la época de secas y lluvias. Se concluye que Oreochromis aureus es una especie omnívora con preferencia hacia el detritus y los restos de plantas vasculares. La alimentación esta determinada por la abundancia de las presas en el medio y en los adultos se basa preferentemente en el detritus. La alimentación no es un factor que limite el desarrollo de la tilapia en la presa Infiernillo.

\section{REFERENCIAS}

Anónimo. 1998. Diagnóstico socioeconómico pesquero de la presa Lic. Adolfo López Mateos Infiernillo, para la elaboración de la propuesta de Norma Oficial Mexicana. Secretaría del Medio Ambiente, Recursos Naturales y Pesca, México. 69 p.

Bagenal, T.B. \& F.W. Tesch. 1978. Age and Growth, p 98 130. In T. Bagenal (ed.). Methods for assessment of fish production in freshwater. Blackwell, Londres.

Barnes, R. 1977. Zoología de los Invertebrados. Ed. Interamericana, México. 826 p.

Bernal, 3.F. 1984. Análisis de los factores relacionados con la producción pesquera de Tilapia nilotica en la presa Adolfo López Mateos (Infiernillo), Mich.-Gro. Tesis de Licenciatura, Universidad Nacional Autónoma de México, México.

Casas, V.M. \& P.E. Benítez 1976. Análisis y normalización de métodos de colecta de parámetros biológicos. Memorias del Simposio de Pesquerías en Aguas Continentales. Tuxtla Gutiérrez, México. 621 p.

Cortés, A.R. \& J.L. Arredondo. 1976. Contribución al estudio limnobiológico de la presa "El Infiernillo" Michoacán-Guerrero. Fideicomiso para el desarrollo de la fauna acuática Serie Técnica (2): 1-29

Guzmán, U.A.1994. Parámetros biológicos de la tilapia (Oreochromis aureus Steindachner, 1864) de la presa Adolfo López Mateos "El Infiernillo", Michoacán-Guerrero, México. Tesis de Licenciatura, Universidad Nacional Autónoma de México, México.

Hyslop, E.J. 1980. Stomach contents analysis a review of methods and their application. J. Fish Biol. 17: 411429.

Jiménez, Q.C., C. Ramírez, N. Hernández \& S. Sabanero. 1994. Informe final del proyecto: Estudio hidrobiológico de la presa Adolfo López Mateos (Infiernillo) Mich.-Gro. Documento interno del Instituto Nacional de la Pesca, Michoacán, México. 40 p. 
Jiménez, B.L., P. Trujillo \& C. Ramírez. 1997. Aspectos reproductivos de Oreochromis aureus (Pisces: Cichlidae) de la presa Infiernillo, Michoacán-Guerrero, México. Memorias del V Congreso Nacional de Ictiología. Sociedad Ictiológica Mexicana Asociación Civil, Mazatlán, Sinaloa, México. 170 p.

Jiménez, B.L. 1999. Análisis de la pesquería de tilapia Oreochromis spp. (Pisces: Cichlidae) en la presa Adolfo López Mateos, Michoacán-Guerrero. Tesis Doctoral, Instituto de Ciencias del Mar y Limnología, México.

Juárez, P.R. 1989. Presa Adolfo López Mateos El Infiernillo Caracterización. Informe preliminar. Secretaría de Pesca, México. 62 p.

Martínez, T.M. 1983. Contribución al conocimiento de la biología de Poecilia sphenops valenciennes (Pisces Poeciliidae), en la presa Zicuirán, Mich., Tesis de Licenciatura, Universidad Michoacana San Nicolás de Hidalgo, Morelia, Michoacán, México.

Mc Bay, L.G. 1961. The biology of Tilapia nilotica Linnaeus, recently redescribed as $T$. aurea Steindachner. Proceedings of the Fifteenth Annual Conference Southeastern Association of Game and Fish Commissioners. 208-218.

Morales, A. 1974. El cultivo de la tilapia en México. Datos Biológicos. Instituto Nacional de la Pesca, México. $124-125$ p.

Needham, P. 1962. A guide to the study of freshwater biology, Holden., San Francisco. 108 p.

Ortega, M.M. 1984. Catálogo de Algas Continentales Recientes de México. Instituto de Biología. Universidad Nacional Autónoma de México, México. 200 p.

Palacios, S.S. 1995. Estudio biológico pesquero de la tilapia Oreochromis aureus (Steindachner, 1864) en la presa Adolfo López Mateos (El Infiernillo), Michoacán-Guerrero, México. Tesis de Licenciatura, Universidad Nacional Autónoma de México, México.

Pennak, W.R. 1978. Fresh-water Invertebrates of the United States. Wiley, Nueva York.

Pinkas, L., M. Oliphant \& I. Iverson. 1971. Food habits of albacore, bluefin tuna and bonito in California waters. Calif. Fish Game (152): 1-105.
Romero, A.C. \& A. Orbe. 1988. Análisis de la explotación pesquera en la presa Lic. Adolfo López Mateos, Mich.-Gro. Período 1981-1986, p 71-89 In Anónimo. Informe de Labores 1986-1988. CRIP-Pátzcuaro. Instituto Nacional de la Pesca. Michoacán, México.

Rosas, M. M. 1976. Sobre la existencia de un nemátodo parásito de tilapia nilotica (Goezia spp. Goeziidae). Memorias del Simposio sobre pesquerías en aguas continentales. Tuxtla Gutiérrez, México: 239-270.

Saiki, K. M. 1976. Mean Bulk Index Method of Fish Food Analysis. Progress. Fish Cult. 38: 55-56.

Salvadores, B.L. 1980. Estudio de la biología y aspectos poblacionales de la tilapia (Sarotherodon aureus Steindachner, 1864) (Pisces: Cichlidae) en la presa "Vicente Guerrero", Gro. México. Tesis de Licenciatura. Facultad de Ciencias. Universidad Nacional Autónoma de México. México:

Salvadores, B.L. \& M. Guzmán. 1983. Contenido estomacal de la tilapia Sarotherodon aureus Steindachner, en la presa "Vicente Guerrero", Guerrero, Edo. de México, México. Biotica 8: 59-70.

Spataru, P. \& M. Zorn, 1978a. Some aspects of natural feed and feeding habits of Tilapia galilaea (Artedi) and Tilapia aurea Steindachner in lake Kineret. Bull. Fish Cult. Israel Bamidgeh 28: 12-17.

Spataru, P. \& M. Zorn. 1978b. Food and feeding habits of Tilapia aurea (Steindachner) in Lake Kinneret (Israel). Aquaculture 13: 67-79.

Windell, J.T. \& S.H. Bowen. 1978. Methods for study of fish diets based on analysis of stomach contents, p. 215-226. In T. Bagenal (ed.). Methods for assessment of fish production in freshwater. Blackwell, Londres.

Wootton, R.J. 1990. Ecology of Teleost Fishes. Chapman and Hall. Fish and Fisheries Series 1. Londres. $404 \mathrm{p}$.

Yañez, A.A., G. Curiel \& V.L. Yañez. 1976. Prospección biológica y ecológica del bagre marino Galeichthys caerulescens (Gunther) en el sistema lagunar costero de Guerrero, México (Pisces: Ariidae). Anales del Centro de Ciencias del Mar y Limnología, Universidad Nacional Autónoma de México, México 3:125-180 